\title{
Development Prototype Design of Virtual Assembly Application-Based Leap Motion ${ }^{\circledR}$
}

\author{
Arif Krisbudiman ${ }^{1}$, Gandjar Kiswanto ${ }^{2}$ \\ ${ }^{1}$ Engineering Staff, Gedung Teknologi II No 250-251, Kawasan PUSPIPTEK, Tangerang Selatan, Banten - 15314, Indonesia \\ ${ }^{2}$ Lecturer, Department of Mechanical Engineering, Universitas Indonesia, Kampus Baru UI - Depok, Indonesia
}

\begin{abstract}
Innovation in design engineering practice is very important in the world of manufacturing in the increasingly competitive global market. Prototyping and evaluation measures are inseparable from the design process in the manufacture of a product. And made one of many physical prototypes require very expensive and time consuming, so the technology of Virtual Reality (VR) is needed, so the industry can quickly and precisely in the decision. VR technology combines a human being with a computer environment visually, touch and hearing, so that the user as if into the virtual world. The goal is that users with hand movements can interact with what is displayed on the computer screen or the user can interact with the environment is unreal to be added into the real world. VR is required for simulations that require a lot of interaction such as prototype assembly methods, or better known as the Virtual Assembly. Virtual Assembly concept which was developed as the ability to assemble a real representation of the physical model, the $3 D$ models in CAD software by simulating the natural movement of the human hand. Leap Motion (accuracy of $0.01 \mathrm{~mm}$ ) was used to replace Microsoft's Kinect (accuracy of 1.5cm) and Motion Glove with flex sensors (accuracy of $1^{\circ}$ ) in several previous research. Leap mot ion controller is a device that captures every movement of the hand to then be processed and integrated with $3 D$ models in CAD software. And simulation of assembly process virtually in CA D software with hand gestures detected by the leap mot ion, assembly parts can be driven either in translation or rotation, zooming and adding the assembly constraint. It also can perform mouse functions (such as left-click, middle-click, right-click and move the mouse cursor position) to a virtual assembly process simulation on CAD software.
\end{abstract}

Keywords-3D CAD Model, API, leap motion controller, virtual assembly, virtual reality

\section{INTRODUCTION}

Innovation is very important for companies to be successful in today's global marketplace. Competitive advantage can be achieved either by doing a revolution in design engineering practice. It must cover all aspects of the design of a product (such as ergonomics, manufacturing, maintenance, product lifespan, etc.) during the early stages of product manufacture. Prototyping and evaluation measures are inseparable from the process of design or manufacture of the product. Although the computer simulation modelling practices such as CAD is currently widely used in the different stages (eg FEA simulation software), makes one of many physical prototypes needed a very expensive and long time. The new

technology is needed so that the industry can quickly and precisely in the decision. The concept of virtual reality (VR) has grown over (State the objectives of the work and provide an adequate background, avoiding a detailed literature survey or a summary of the results) the past two decades. VR has changed the way engineers and scientists to the computer to perform mathematical simulations, data visualization, and decision making (Bryson, S., 1996; Eddy, J. and Kemper E., Lewis, 2002; Xianglong, Y., Yuncheng, F., Tao, L. and Fei, W., 2001; Zorriassatine, F., Wykes, C., Parkin, R. and Gindy, N., 2003). VR technology combines multiple human environments with a computer to provide a variety of sensations (visual, haptic, auditory, etc.) that makes the user as if into the virtual world. It allows the user to natural human movement can interact with what is displayed on the computer screen. The aim is that the user can interact with the virtual environment you want to create or add in the real world. This makes it the perfect tool for the VR simulation activities that require a lot of user interaction such as prototype assembly methods, or better known as the virtual assembly. Jayaram et al., in 1997, a virtual assembly defines as "the use of computer tools to make or assist the engineers to take decisions in the assembly through analysis, predictive modelling, visualization, and presentation of data without any physical product or process support tool significantly" (Jayaram, S., Connacher, Hugh I. and Lyons Kevin W., 1997). This definition emphasizes the use of computer equipment and visualization techniques that facilitate decision-making in the assembly process with the addition of the virtual environment to the real interaction as an integrated part of the virtual assembly simulation. 
Then the definition by Kim and Vance in 2003, described the virtual assembly is "the ability to assemble CAD models of components in 3D, user environment and natural human motion" (Kim Chang E. and Vance, Judy M., 2003). This illustrates that the incorporation of 3D environments, VR and natural human interaction are an important part of the virtual assembly. Virtual assembly definition has evolved with VR technology, after the visualization problems can be solved, a new definition is added natural human interaction as a challenge to the virtual assembly simulation. And the development of VR technology is more advanced then expands previous definitions to provide a more comprehensive picture of the virtual assembly.

Virtual assembly in th is paper is defined as the ability to assemble a real representation of the physical model, the 3D models in CAD through a realistic simulation of the environment as the natural movement of the human hand in the assembly process. Planning the assembly process is an important step in the development of products which includes details of assembly operations that describes the steps the most quickly, accurately and efficiently. And the cost of the assembly process is often a large part of the total cost of the product (Boothroyd, G. and Dewhurst, P., 1989). Thus, it is very important to develop proper assembly in the initial design planning stages. A good assembly plan by combining consideration for minimum assembly time, low cost, ergonomics and operator safety. And the design of the assembly process that will either be able to imp rove the efficiency, quality, reduce costs and shorten product time to market.

Expert assembly planners is still using the traditional approach in which they have to see the 3D model in a CAD of parts to be assembled on a 2D screen of a computer to check the part geometry and determine the assembly sequence for the new product. Another method for planning the assembly, including doing some experiments with a physical prototype assembling and find the best sequence of assembly. This causes the assembly becomes more complicated tasks, such methods tend to be more time-consuming, expensive and prone to errors.

Planning assembly with the aid of computer simulations became popular in solving existing problems. Much research has been done to develop an algorithm to generate a suitable sequence assembly process or the most optimal. But there are some things that still need to be addressed, because of the increased number of components, the assembly sequence increases exponentially making it difficult to characterize the criteria in selecting the most appropriate assembly sequence for specific products (Dewar, R. G., Carpenter, I. D., Ritchie, J. M. and Simmons, J. E. L., 1997).
Modern CAD systems also provide the ability to build a CAD assembly model. However, CA D systems are still using $2 \mathrm{D}$ environments such as a keyboard and mouse as well as the user must manually choose the surface, axis lines or the edges of the components to be assembled. Thus it does not reflect the human interaction with the component parts of complex shape. And it becomes difficult to predict what will appear either in the process of assembly, maintenance or during operation, for example, ensuring accessibility to replace components during maintenance and the effect of changing the assembly sequence. It is also in addressing issues related to ergonomics as the ease in the assembly process.

VR technology plays an important role in the simulation of the interaction between humans and computers in 3D by making as if the user is logged into the virtual world displayed on the computer screen. Simulating a virtual assembly process is to produce the most suitable process in assembling a product so as to reduce the high cost and time required for the process of making physical prototypes.

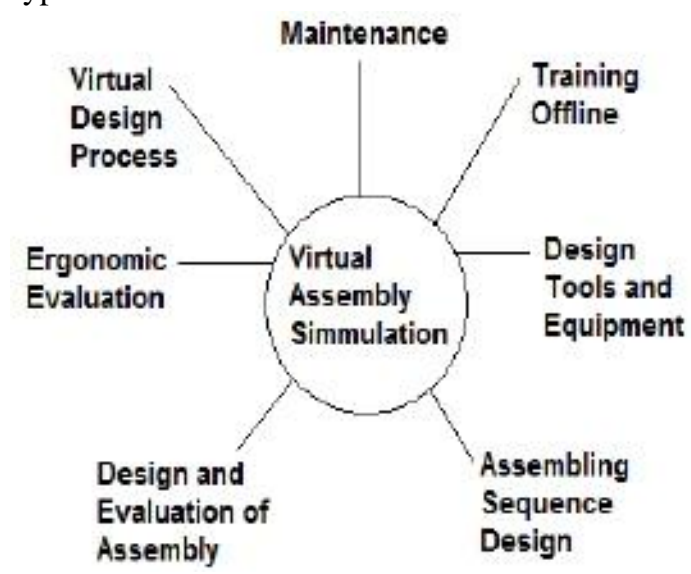

Fig.1: Application of virtual assembly simulation

Using VR technology, the simulation can be done and different assembly sequences can be analyzed by utilizing existing CAD data. The ability of the virtual assembly can be applied to produce a competitive product designs in various aspects, such as process design, ergonomics, workstation layout, assembly design, fixtures and equipment, training, and maintenance (Figure 1).

This study combines motion detection technique hand to interact with 3D models in CAD software in its application to the virtual assembly. And approaches for detecting the movement is with method System Machine Vision (SMV) and image processing techniques. In recent years there are some inexpensive sensor devices such as Microsoft's Kinect $(1.5 \mathrm{~cm}$ accuracy) and Leap Motion (accuracy $0.01 \mathrm{~mm}$ ), resulting in a virtual assembly has grown not only limited to using a mouse, keyboard and touchscreen. There was also a motion that uses the virtual assembly $g$ 
love with flex sensors (accuracy of $1^{\circ}$ ) in interaction with 3D models in CAD software. And in this study used a leap motion which has a higher degree of accuracy and include Software Development Kit (SDK) and Application Programming Interface (API) for interacting with other applications, making it possible to simulate a virtual assembly process.

\section{METHODOLOGY/ EXPERIMENTAL}

The method used in the design of a prototype application is to use a Virtual Assembly leap motion controller as a detector of hand movements. And by utilizing the SDK of leap motion and API Object Model from Autodesk Inventor, created and developed a prototype design of the application of Virtual Assembly by using software, namely Microsoft Visual Studio Professional 2013, to simulate assembly in CAD software, the Autodesk Inventor Professional 2014 - Student Version (Figure 2).

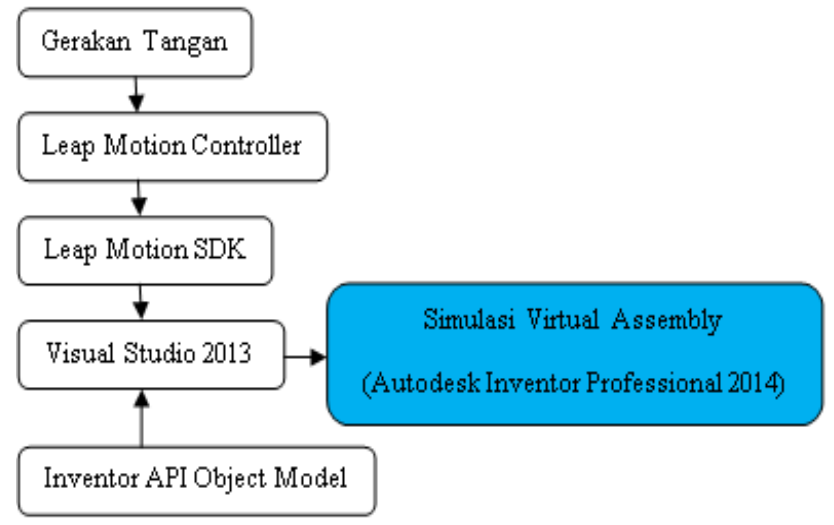

Fig.2: Conceptual design of the virtual assembly system

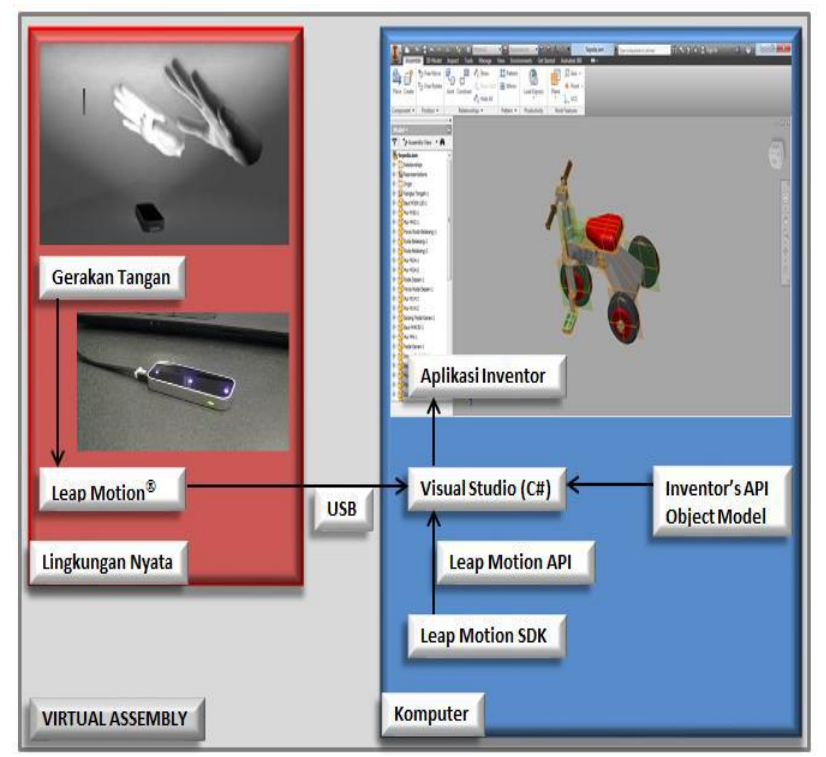

Fig.3: Virtual Assembly System Design

Leap hand gestures detected by the motion controller and the main data used are the position and movement of the point of the finger (finger positioning). The data will be integrated with assembly parts in the application of
Autodesk Inventor, use other applications built with $\mathrm{C}$ \# programming language to software Visual Studio 2013. The application was made by utilizing Leap Motion SDK and Inventor's API Object Model so as to relate the data from leap mot ion with Data 3D CAD models from Autodesk Inventor application. The technical specifications of the computer used is as follows:

1. Processor: Intel (R) Core (TM ) i5-3210M CPU @ $2.50 \mathrm{GHz}$

2. RAM: $8.00 \mathrm{~GB}$

3. System type: 64-bit Operating System (Windows 7 Home Basic).

In the simulation of assembly process virtually in Autodesk Inventor application is divided into four major parts, namely:

1. Moving parts assembly both translational

2. Move the rotation assembly, both parts

3. Zoom in or out assembly part (zooming process)

4. Adding assembly constraints.

And the process can be run with the command through gestures or called as a command gesture. Command hand movements may also perform mouse functions, such as: left-click, middle-click and right-click and move the mouse cursor position.

Command hand movements are made, among others:

1. Left-Click: use the right hand with the middle finger, forefinger and thumb open and left-click to do is to close and open the thumb (the tip of the index finger as a mouse cursor position).

2. Middle-Click: use the right hand with the middle finger, forefinger and thumb open and to middle click is to open and close the ring finger (index finger as a mouse cursor position).

3. Right-Click: use the right hand with the middle finger, forefinger and thumb open and to do the right-click with the closing and opening of the middle finger (index finger as a mouse cursor position).

4. Moving in translational assembly parts are selected (Free Move): use the right hand with the middle finger, forefinger and thumb open, then point the mouse cursor to the parts that will be selected to be moved. Then close the thumb to select (left-click) and the selected part can be moved in accordance with movement of the hand movement.

5. Moving in translational all assembly parts simultaneously (Pan): use the right hand with the middle finger, forefinger and thumb open, then middleclick to open the command ring finger. After that all assembly parts can be driven simultaneously in accordance with the displacement movement of the hand.

6. Moving in rotation (Orbit View): use the right hand with the index finger, middle finger, ring finger and 
little finger open. After that all assembly parts can be driven at the same rotation in accordance with the movement of the hand.

Zoom in or out (zooming process): use the right hand with the thumb open. After that hand movement away from the user to zoom out and approaching from the user to zoom all the assembly parts.

The steps undertaken in the development of prototype design application-based Virtual Assembly leap motion, as follows:

1. Making an application with Visual Studio using C \# programming language to connect to leap motion controller.

2. The application development with Visual Studio using $\mathrm{C}$ \# programming language to read data from the leap mot ion movement of the hand.

3. Replace the mouse cursor position with the position of the user's finger is detected by the motion leap.

4. Create a movement of the hand which can perform certain commands, and replace the function of the mouse (left-click, middle-click and right-click).

5. Integrate data from leap motion with the 3D model data in CAD software, the Autodesk Inventor.

6. Creating a movement that can perform certain commands on CAD software (command gesture).

7. Creating a Graphic User Interface (GUI) to display related information in performing a virtual assembly process simulation using motion leap in CAD software. In Figure 4 above can be seen information obtained from the leap motion, such as: the position of the fingers, hand movements (pitch, roll and yaw), number of hands (right or left), the number of tools and the type of gesture. And other information that informs the assembly document on the application of Autodesk Inventor have been opened beforehand, while in the LOG contains real time information to the commandments of what has been done.

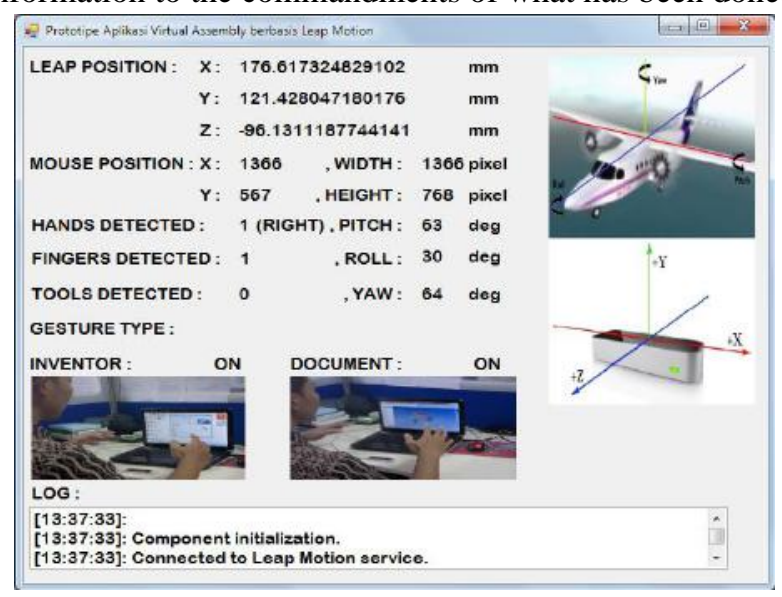

Fig.4: Graphic User Interface

Commands include: Left-Click, Middle-Click, RightClick, Free Move, Pan, Orbit View, Zoom In, Zoom Out and Assembly Constraint.
Development of Virtual Assembly prototype design applications using procedures and algorithms are programmed using the programming language $\mathrm{C} \mathrm{\# .}$ Algorithms and procedures are as follows:

1. Connection to leap motion controller

\section{algorithm:}

using Leap.dll

New controller

Initialization controller

Connection controller

Turn all gestures (circle, key tap, tap and swipe the screen)

The reading of the data of each frame of a leap motion

\section{procedure:}

a. Make sure the leap motion controller is connected to a computer through a USB port

b. Make sure the indicator light from the flame leap motion has been colored green, orange if service mark of leap motion and the road yet to run: on the list view select Leap Services $\rightarrow$ Service $\rightarrow$ click Start.

c. Three files, namely: Leap.dll, Leap CSharp.dll and Leap CSharp.NET4.0.dll must copy first into the project folder (PROJECT $\rightarrow$ Add Existing Item ... or Shift + Alt + A).

d. Leap CSharp.NET4.0.dll should be inserted first into the reference project (PROJECT $\rightarrow$ Add Reference ...).

2. Reading the data of hand gestures

\section{Algorithm:}

Reading data from the previous frame of leap motion The reading of the current data frame of leap motion

Reading the data of hand from leap motion

Reading the data of gesture from leap motion

\section{Procedure:}

a. Reading data for each frame hand is detected by motion leap.

b. Data reading gesture was to some 10,000 frames per second which is detected by the motion leap.

3. Data reading position of the finger

\section{Algorithm:}

The reading of the data of each frame from leap motion

Reading the data of hand-motion from leap motion

Reading the data of finger-motion from leap motion

\section{Procedure:}

a. Reading the data of right hand fingers, if only detected a right hand

b. Reading the data of left hand fingers, if only detected a left hand alone. 
c. Reading the data of right hand fingers, if it is detected more than one hand.

4. The position of the fingers used as the position of the mouse cursor

\section{Algorithm:}

Reading the data of each frame from leap motion Reading the data of hand-motion from leap motion Reading the data of finger-motion from leap motion Using user32.dll

Setting the mouse cursor position $(\mathrm{X}, \mathrm{Y})$ with the position of one finger is detected by the motion leap

\section{Procedure:}

a. Setting the mouse cursor position changes if the speed of movement of the fingers (Tip Velocity $>25$ ), to eliminate vibration fingers.

b. Conversion unit of the position of the fingers (mm) into the mouse cursor units (pixels).

5. The command hand movements replace the functions of the mouse (left-click, middle-click and right-click).

\section{Algorithm:}

Reading the data of each frame from a leap motion Reading the data of hand-motion from leap motion Reading the data of finger-motion from leap motion Using user32.dll

Using mouse_event (Move, LeftDown, LeftUp, RightDown, RightUp, MiddleDown, MiddleUp, Xdown, XUP, Wheel, VirtualDesk, Absolute)

Procedure: Changes in the finger motion is read by a determined leap into commands to perform the functions of the mouse (left-click, middle-click and right-click).

6. The integration of data from leap motion with the 3D model data in CAD software.

\section{Algorithm:}

Reading the data of hand-motion from leap motion

Reading the data of finger-motion from leap motion

Using user32.dll

Using reference Autodesk.Inventor.interop

Reading data from Autodesk Inventor 3D models

Procedure: Autodesk.Inventor.interop.dll should be inserted first into the reference project (PROJECT $\rightarrow$ Add Reference ...).

7. Creating a movement that can perform certain commands on CAD software (command gesture).

\section{Algorithm:}

Reading the data of hand-motion from leap motion

Reading the data of gesture from leap motion

Reading the data of finger-motion from leap motion

Using user32.dll

Using mouse_event (Move, LeftDown, LeftUp,

RightDown, RightUp, MiddleDown, M iddleUp,

Xdown, XUP, Wheel, VirtualDesk, Absolute)

Using reference Autodesk.Inventor.interop
Using class Camera.cs (TranslateView function, ChangeView and Zoom)

Procedure: Hand movements are read by leap motion was determined to be a command to perform certain commands on the application of Autodesk Inventor (Free Move, Orbit View, Pan, Zoom and insert assembly constraints).

8. Creating a Graphic User Interface (GUI).

\section{Algorithm:}

Display design (layout) GUI desired

Reading the data of hand-motion from leap motion

Reading the data of gesture from leap motion

Reading the data of finger-motion from leap motion

Reading data from Autodesk Inventor

Displaying all desired data as output information

Procedure: Data from the leap motion with regard to the position of the fingers, hand movements (pitch, roll and yaw), number of hands (right or left), the number of tools and the type of gesture and information whether the leap motion controller has been connected properly to the computer before the application of Virtual Assembly run.

Data from Autodesk Inventor related to the commands that are being done on CAD software, such as: Left Click, Middle Click, Right Click, Free Move, Pan, Orbit View, Zoom In, Zoom Out and Assembly Constraint and information whether the document assembly has been opened before the application is run Virtual Assembly.

\section{RESULTS}

Virtual simulation experiment Assembly is done by using multiple assembly files (*.Iam), which can be divided into:

1. Assembly file 1 , consists of 5 parts

2. Assembly file 2, consists of seven parts

3. Assembly file 3, consists of nine parts

Virtual assembly process simulation in Autodesk Inventor using motion leap and applications made, among others:

1. Replacing a mouse function, namely move the mouse cursor position with hand gestures detected by the motion leap. The movement is useful for directing, selecting and moving parts in translation in a virtual simulation of assembly process.

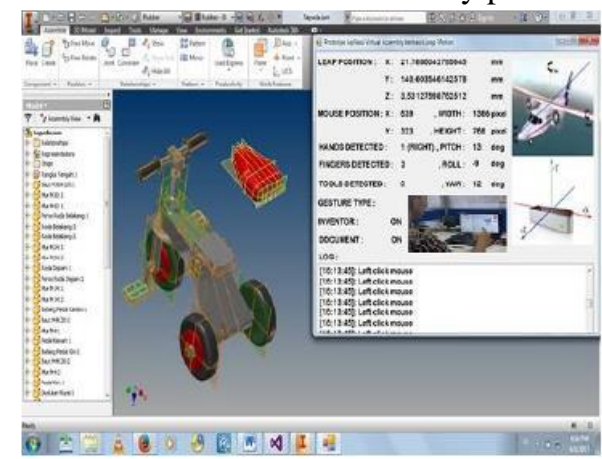




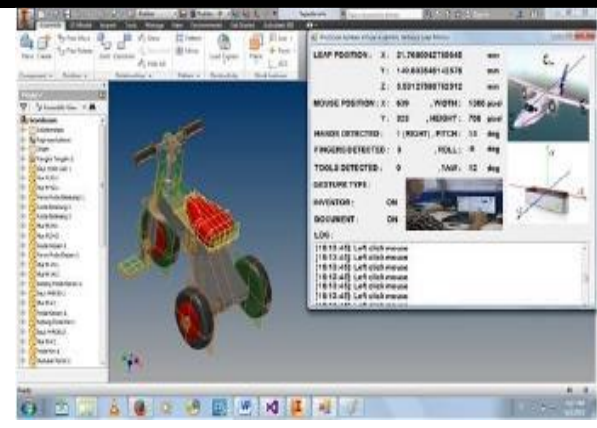

Fig.5: Command left click mouse

2. Replacing a mouse function, that is to left-click, middle-click and right-click. The hand gesture commands useful for selecting and moving parts in the translation.

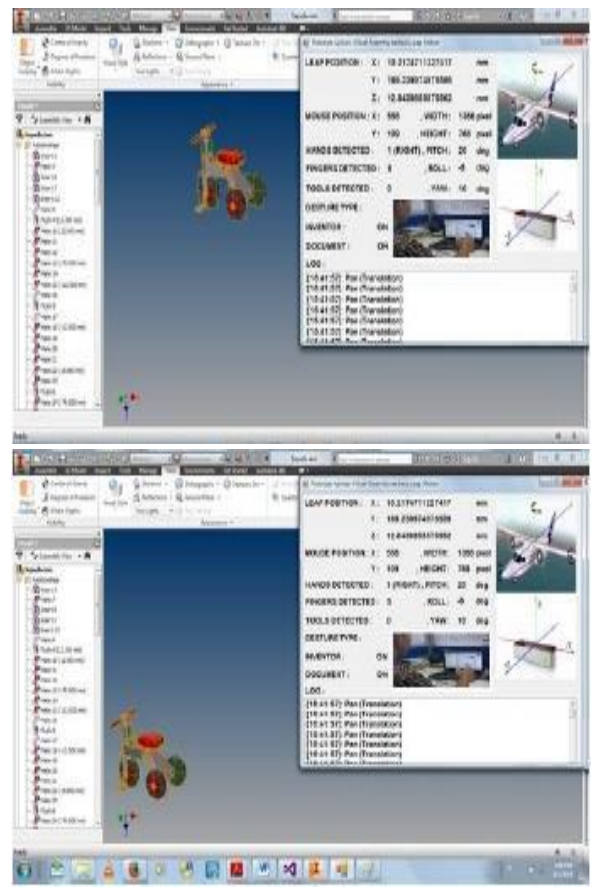

Fig.6: Command Pan (Translation)

3. Mobilize assembly part in translation and rotation with command hand movements in a virtual simulation of assembly process.

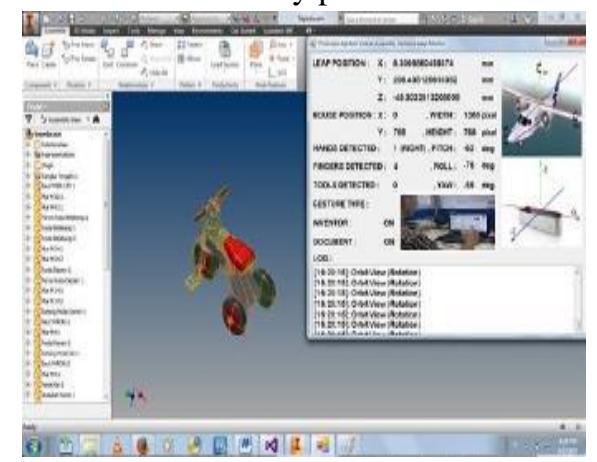

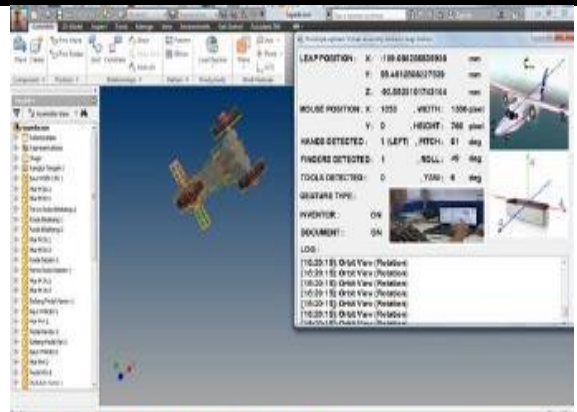

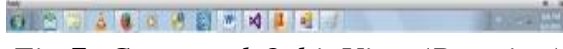

Fig.7: Command Orbit View (Rotation)

4. Zoom in or out (zooming process) of the assembly part

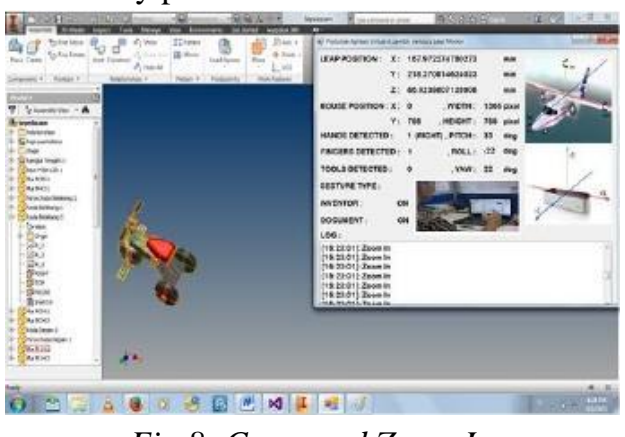

Fig.8: Command Zoom In

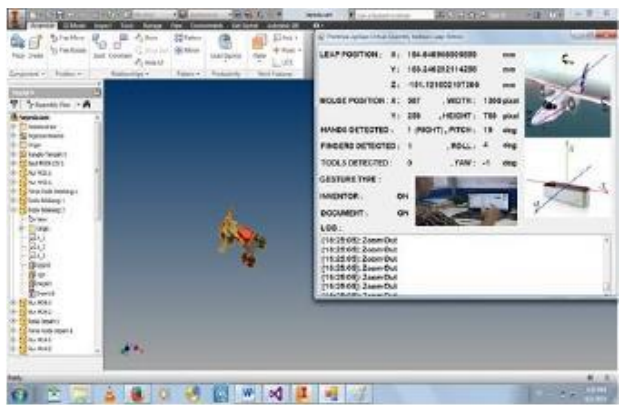

Fig.9: Command Zoom Out

5. Adding assembly constraints between two parts for virtual assembly process.

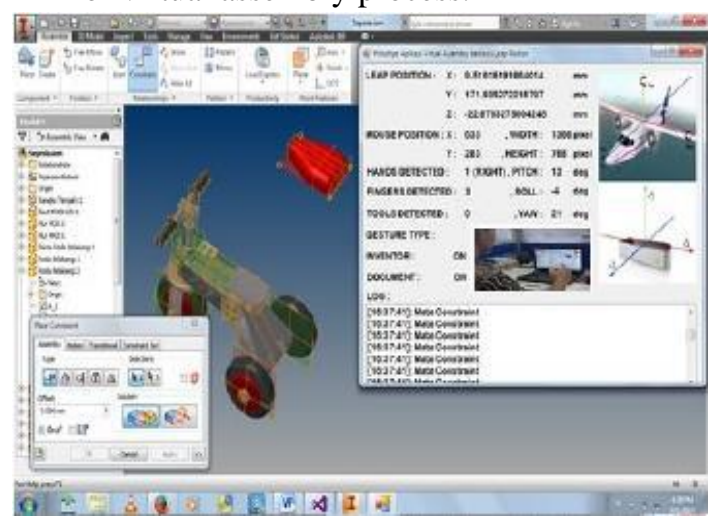




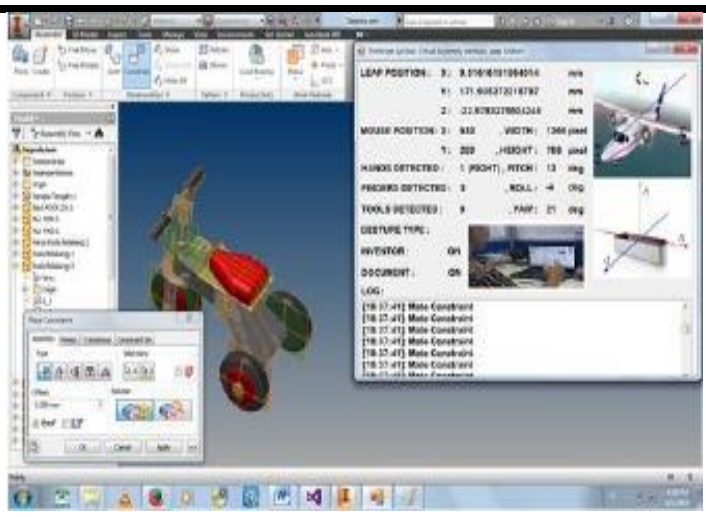

Fig. 10: Command Mate Constraint

\section{DISCUSSION}

Based on the simulation results that the assembly part in Autodesk Inventor can move, enlarged and reduced by using a hand gesture commands are detected by a motion leap. As for adding assembly constraints still use the "Place Constraint" in Autodesk Inventor, but still uses the motion leap-click menu and selection of spare parts assembly (surface, edge or axis) which will be used as constraints. This was due not yet succeeded in choosing the assembly spare parts directly using motion leap through hand motions, because the function of the selection of spare parts of the API can only be used with a mouse (as the standard default). And simulations were done using different assembly file, shows that with more and more part in the assembly file the movement more visible dashed.

\section{CONCLUSION}

Based on the research results can be concluded as follows:

1. Data reading leap motion (movement of the hand, the finger position and gesture) through other applications that are programmed in $\mathrm{C} \#$ has been successfully carried out.

2. Through the development of a prototype application that created virtual assembly has managed to perform the command function mouse (left-click, middleclick, right-click and move the mouse cursor position).

3. Based on the virtual assembly simulation done by hand movement has succeeded in moving the assembly part by translation, rotation, zoom and adding assembly constraint.

4. In adding assembly constraints still use the menu on the Autodesk Inventor leap motion due to limitations in choosing the parts section directly by using the command hand movements.

\section{ACKNOWLEDGEMENTS}

For research on Virtual Assembly theme in the world of manufacturing, the authors suggest the following research:
1. Virtual Assembly technology combined with Augmented Reality (AR).

2. The use of more than one leap motion with the program algorithms to simulate a Virtual Assembly.

3. Selection of spare parts directly using hand gestures via motion leap can be made in virtually any assembly process simulation on CAD software.

\section{REFERENCES}

[1] Boothroyd, G. and Dewhurst, P., (1989), Product Design for Assembly. New York: McGraw-Hill, Inc.

[2] Bryson, S., (1996), Virtual Reality in Scientific Visualization, vol. 39, pp. 62-71, Communications of the ACM.

[3] Dewar, R. G., Carpenter, I. D., Ritchie, J. M. and Simmons, J. E. L., (1997), Assembly Planning in a Virtual Environment, presented at Proceedings of Portland International Conference on management of Engineering and Technology (PICMET 97), Portland, OR.

[4] Eddy, J. and Kemper E., Lewis, (2002), Visualization of Multidimensional Design and Optimization Data using Cloud Visualization, ASME Design Engineering Technical Conferences and Computers and Information in Engineering Conference (DETC2002/DA C-34130), Montreal, Canada.

[5] Jayaram, S., Connacher, Hugh I. and Lyons Kevin W., (1997), Virtual Assembly using Virtual Reality Techniques, Computer Aided Design, vol. 29, pp. 575-584.

[6] Kim Chang E. and Vance, Judy M., (2003), Using Vps (Voxmap Pointshell) As The Basis For Interaction in a Virtual Assembly Environment, ASME Design Engineering Technical Conferences and Computers and Information in Engineering Conference (DETC2003/ CIE-48297), Chicago, IL.

[7] Xianglong, Y., Yuncheng, F., Tao, L. and Fei, W., (2001), Solving Sequential Decision-making Problems Under Virtual Reality Simulation System, Winter Simulation Conference Proceedings, Arlington, Virginia.

[8] Jaiswal, M. (2014). Fundamental of Interactive Computer Graphics and Quality Assessment. Journal Of Emerging Technologies And Innovative Research, $1(5)$.

[9] Zorriassatine, F., Wykes, C., Parkin, R. and Gindy, N., (2003), A Survey of Virtual Prototyping Techniques for Mechanical Product Development, Institution of Mechanical Engineers, Part B: Journal of Engineering Manufacture, vol. 217, pp. 513-530. 